\title{
Menstrual Health: What Have We Learned?
}

\author{
Nimble O J' ${ }^{1}$ Bibil Babu $\mathbf{C}^{2}$ \\ ${ }^{1}$ Assistant Professor, Centre for Management Studies, Presidency College, Kempapura, Hebbal, Bangalore, \\ ${ }^{2} P G$ Student Dept. of Oral \& Maxillofacial Pathology Royal Dental College Palakkad
}

\begin{abstract}
Women specific health issues are always neglected and underestimated by the society including women themselves. Premenstrual syndromes (PMS), Thyroid issues, Post-partum depression are common cause of physical, psychological and social problems in women of reproductive age.

This research explores the evidence about women's awareness and attitude about menstrual health related problems and alternative and sustainable menstruation practices. This study was designed to explore the potential health physical and environmental benefits that can accrue by women with safe, affordable, and convenient materials, such as the menstrual cup, for menstrual management. Researcher collected primary data from 28 women using convenience sampling technique to understand awareness and attitude about women health issues. Researcher also collected primary data from 50 women using cluster sampling method to investigate attitudes and awareness towards sustainable menstruation practices and to an alternative menstrual product. The result showed positive reactions to an alternative menstrual product
\end{abstract}

Key words: PMS, Thyroid, Menstrual cup, sustainability, environmental hazards, health hazards(JEL Classification Code: Q01, Q53, I18, I19)

\section{Introduction}

From the ancient times women menstrual health related problems are considered not to be discussed and disclosed to others. Society, especially in developing countries, considers these problems as internal concerns of women and never allowed to discuss. Menstrual disorders are problems related to a woman's normal menstrual cycle which can disrupt a woman's daily life. Recently, with the effect of globalisation and quality of education, people started discussing and anlalysing these issues.

Premenstrual syndrome (PMS) is a combination of symptoms that many women get about a week or two before their Menstrual Cycle. It is always misinterpreted with premenstrual symptoms and undermined by the society, especially women. These symptoms are varied from each woman. Medically the reasons for these problems are unknown, but it is proved that after ovulation, ie; mid of menstrual cycle, Estrogen and Progesterone levels falls and which affects Serotonin
Level. Serotonin Level is a brain chemical affects mood, sleeping pattern and appetite. This creates mood swings and other behavioral problems for women. Many scholars believe that, PMS can occur due to maternal history and hereditary.

\section{Plethora of Symptoms}

Symptoms of PMS are divided into two

\section{Physical}

- Abdominal bloating

- Constipation and diarrhea

- Breast tenderness or swelling

- Joint or muscle painHeadache, migraine and sensitivity

- Acne, Tiredness and poor sleep or sleepiness 


\section{Psychological}

- Anxiety and confusion, feelings of loneliness and paranoia

- Depression and lowered mood, which may include suicidal thoughts

- Difficulties concentrating,

- Drop in self-esteem and confidence, Drop in sexual desire, or (occasionally) an increase

- Irritability, Mood swings, weepiness.

PMSworsen during pre-menopause and it is also connected with mid-life syndrome. Severe form is known as premenstrual dysphoric disorder (PMDD)

\section{Thyroid and Women Health}

Thyroid condition is another women centric problem, even though it is common with men and children. A woman with thyroid issues can develop serious menstrual related problems. Researchers found that, women have 5 to 8 times more likely to be diagnosed with Thyroid. Thyroid develops due to hormonal imbalances. Hormones are chemicals that are released to the blood streams which regulates specific body functions. Thyroid gland is a part of our body's endocrine system which produces 2 hormones, Thyroxine T4 and Thyronine T3.

\section{Types Thyroid Disease}

- Hyperthyroidism (overactive thyroid) occurs when your thyroid gland produces too much of the hormone thyroxine.

- Hypothyroidism (underactive thyroid) is a condition in which your thyroid gland doesn't produce enough hormones.

Symptoms of Hypothyroidism

- Fatigue, Slowed Heart Rate

- Depression

- Constipation
- $\quad$ Feeling Cold

- Dry Skin, Weight Gain

- Muscle Weakness, Pain And Stiffness

- Dry, Thinning Hair

- Fertility Difficulties Or Menstrual Changes

Symptoms of Hyperthyroidism

- Unintentional weight loss

- Irregular heartbeat

- Nervousness, anxiety and irritability

- Increased sensitivity to heat

- Changes in menstrual patterns

- An enlarged thyroid gland

- Fatigue, muscle weakness, Difficulty sleeping

- $\quad$ Skin thinning, Fine, brittle hair

Sustainable and Environmental Friendly Menstrual Practices

Environment provides the input for man's productive activities. The environment provides life-support services such as maintenance of genetic diversity and stabilization of the ecosystem. Protecting environment without disturbing the development is the only way for sustainable development. Waste management is very crucial for sustainable development and Sanitary waste disposal has become an increasing problem in India as the plastic used in disposable sanitary napkins are not bio-degradable and lead to health and environmental hazards.

For years, women have used either tampons or pads to collect blood during menstruation, but a menstrual cup provides women with an alternative to these traditional methods. As the most favoured waste management technique is prevention and minimization, menstrual cup is the best sustainable practice available in the market. 
Although the present research approach this topic from an Indian perspective, because the menstrual cup is a substitute for disposable products and may be sustainable the findings may have implications for women's health internationally.

\section{Literature Review}

\section{Menstrual Health Problems}

Ryu, A., \& Kim, T. H. (2015) ${ }^{12}$ Premenstrual syndrome: A mini review explains PMS scientifically. They recommended a stepwise therapeutic approach to tackle PMS problem. The researchers also suggested lifestyle modification, cognitive behavioral therapy and herbal medicine.

Figert, A. (2017) ${ }^{4}$ in their study, Women and the ownership of PMS: The structuring of a psychiatric disorderanalysed the controversial decision of APA to include PMS into its revised diagnostic manual.

Menstrual-related problems and psychological distress among women in the United States byStrine, T. W., Chapman, D. P., \& Ahluwalia, I. B. $(2005)^{13}$ analysedthe association of menstrual related problems with mental health and health behaviours. The study suggested health providers to examine mental health concerns of women, because menstrual related health issues creates problem for public health.

Banerjee N, Roy KK, TakkarD $(2000)^{2}$ in their study, Premenstrual dysphoric disorder--a study from India, examined the prevalence of PMDD among Indian women. They used structured performa to record PMDD symptoms over two menstrual cycles and identified the prevalence of the condition among participants.

Raval, C. M., Panchal, B. N., Tiwari, D. S., Vala, A. U., \& Bhatt, R. B. (2016) ${ }^{10}$ in their research, Prevalence of premenstrual syndrome and premenstrual dysphoric disorder among college students of Bhavnagar, Gujarat done a cross sectional study in five college in Bhavnagar. Girls were analysed sociodemographic data and concluded Prevalence of PMS among college students is similar to other studies from Asia

\section{Sustainable Menstrual Practices}

Meenakshi, N. $(2020)^{6}$ in the research article, Taboo in consumption: Social structure, gender and sustainable menstrual products explored taboos and barriers in consumption choices for sustainable menstrual product. Using qualitative research design the researcher analysed this behavioural pattern.

Green is the new colour for menstruation. Uger fabric pads show the way through a sustainable perspective written by Murthy, L. (2015) ${ }^{7}$, examined menstrual products used by women in South Rajasthan and developed a new product, the Uger fabric sanitary napkin to fill the gaps.

A study into public awareness of the environmental impact of menstrual products and product choice by Peberdy, E., Jones, A., \& Green, D. (2019) ${ }^{9}$, explored the level of awareness people have about the environmental impact of menstrual products. The result showed that most participants were not aware of the environmental impact of these products.

\section{Research Design}

\section{Objectives of the Study}

1. To understand awareness and attitude of women towards menstrual health problems

2. To determine Menstruation cup as sustainable alternative to hazardous menstrual products

3. To assess the awareness, attitude and the practices towards menstruation cup

\section{Research Methodology}

Researchers used exploratory research with qualitative and quantitative variables. This research is based on sample survey with cluster random sampling method. Researcher collected primary data from 28 women using convenience sampling technique to understand awareness and attitude of women in general about women health issues using google forms. Researcher also collected primary data from 50 women using cluster sampling method dividing the sample into 
three clusters of working professionals, home makers and students to investigate attitudes and awareness towards sustainable menstruation practices and to an alternative menstrual product. Collected data was analyseddescriptively using SPSS and Excel software.

\section{Scope of study}

Scope of this study is limited to awareness and attitude of women towards menstrual health problems like PMS and hormonal imbalance issues like Thyroid. Study was on environment and health impact of using commercial women hygiene products and sustainable alternative women hygiene product available in market. Area of study was Bangalore city and overall sample size was limited to 75 .

\section{Analysis: Awareness and Attitude towards} Menstrual Health Problems

Primary data was collected from 28 females of 20 to 45 age category using structured questionnaire. $64 \%$ respondents were 35 to 45 age category, who has highest chance of having PMS, Thyroid and Mid-life crisis. 17\% of the respondents fall under 20 to 30 age group. 53.57\% participants were employed, $14.28 \%$ self-employed and $28.57 \%$ were home makers.

Among the participants, $67.9 \%$ have regular and normal cycles, but unfortunately all of them consider pain,discomfort and mood swings are normal during menstrual cycles. The below figures explains all participants experiences discomforts physically or psychologically during their cycle

Physical and Psychological Problems of women during Menstrual cycle

\begin{tabular}{|c|c|}
\hline Problems & Percentage \\
\hline Abdominal bloating & 35.7 \\
\hline Acne & 28.6 \\
\hline Digestive upsets, including constipation and diarrhea & 10.7 \\
\hline Breast tenderness or swelling & 32.1 \\
\hline Joint or muscle pain & 25 \\
\hline Tiredness and poor sleep or sleepiness & 32.1 \\
\hline Food cravings & 25 \\
\hline Headache, migraine and increased sensitivity to sounds, light and touch & 32.1 \\
\hline Anxiety and confusion, feelings of loneliness and paranoia & 25 \\
\hline Depression and lowered mood, which may include suicidal thoughts & 7.1 \\
\hline Difficulties concentrating, memory lapses & 14.3 \\
\hline Drop in self-esteem and confidence, leading to social isolation & 14.3 \\
\hline Drop in sexual desire, or (occasionally) an increase & 10.7 \\
\hline Irritability, including angry outbursts at personal(specially with husband) and professional life & 32.1 \\
\hline Mood swings, weepiness. & 60.7 \\
\hline Other Problems & 3.6 \\
\hline
\end{tabular}




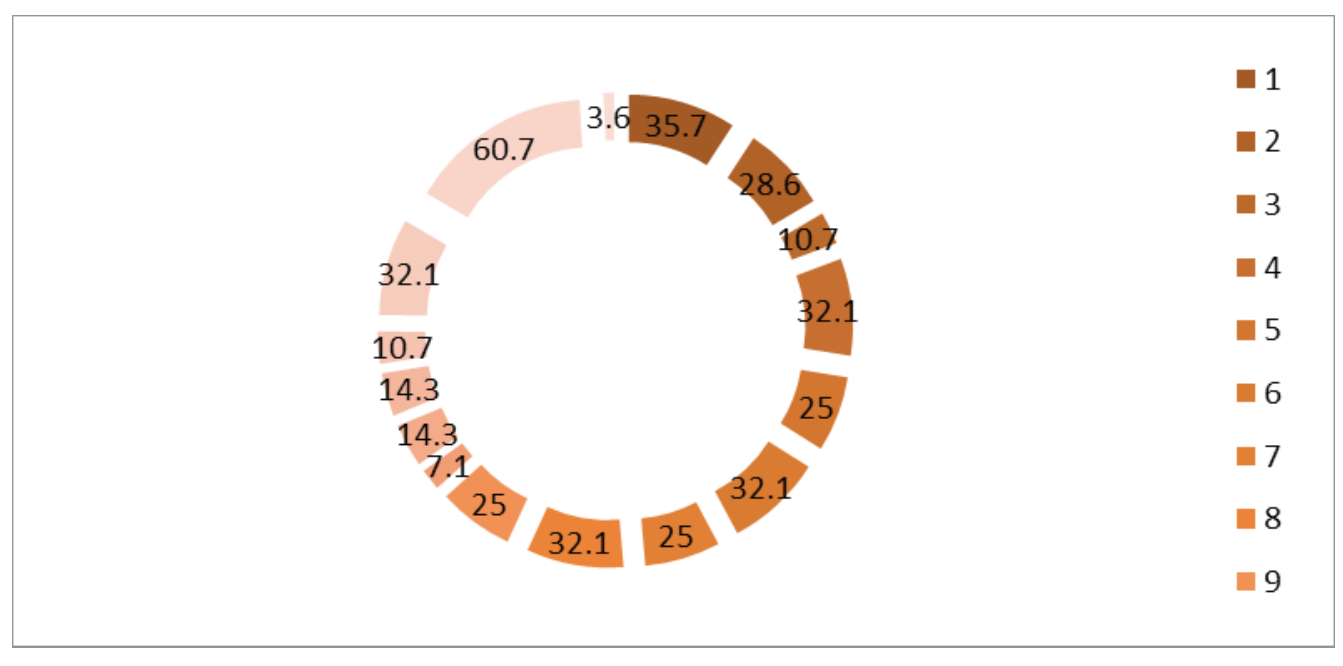

Source:Primary Data collected using structured questionnaire

$71.4 \%$ respondents discuss their menstrual related problems with partner and only $21.4 \%$ uses technology driven apps to track their cycles and related problems. Education and city life culture are the reasons for the respondents to discuss their issues with partner, but most of them are reluctant to use mobile applications because of privacy concerns.

Multiple physical and psychological changes due to changed environment and work stress creates hormonal imbalance among people, which leads to Thyroid problems. Women are more prone to Thyroid. Most of them are unaware of the existence of this condition. $100 \%$ Respondents of the survey are experiencing hypothyroid and hyperthyroid symptoms.

\section{Sustainable Menstrual Practices}

Primary data collected for this research with the structured questionnaires is collected from 50 respondents; among these 5 were eliminated due to inappropriate responses. In this sample size, 20 are working women, 15 house wives and 10 are students

Among the 45 samples collected $42.22 \%$ heard about Tampons, $100 \%$ are aware about the disposable sanitary napkins, $60 \%$ are heard about menstrual cups, $22.22 \%$ about bio degradable napkins and 64.44\% heard about reusable products.Among the respondents, commonly used product during the menstrual cycle is disposable sanitary napkins. Only $6.7 \%$ females are using menstrual cup.

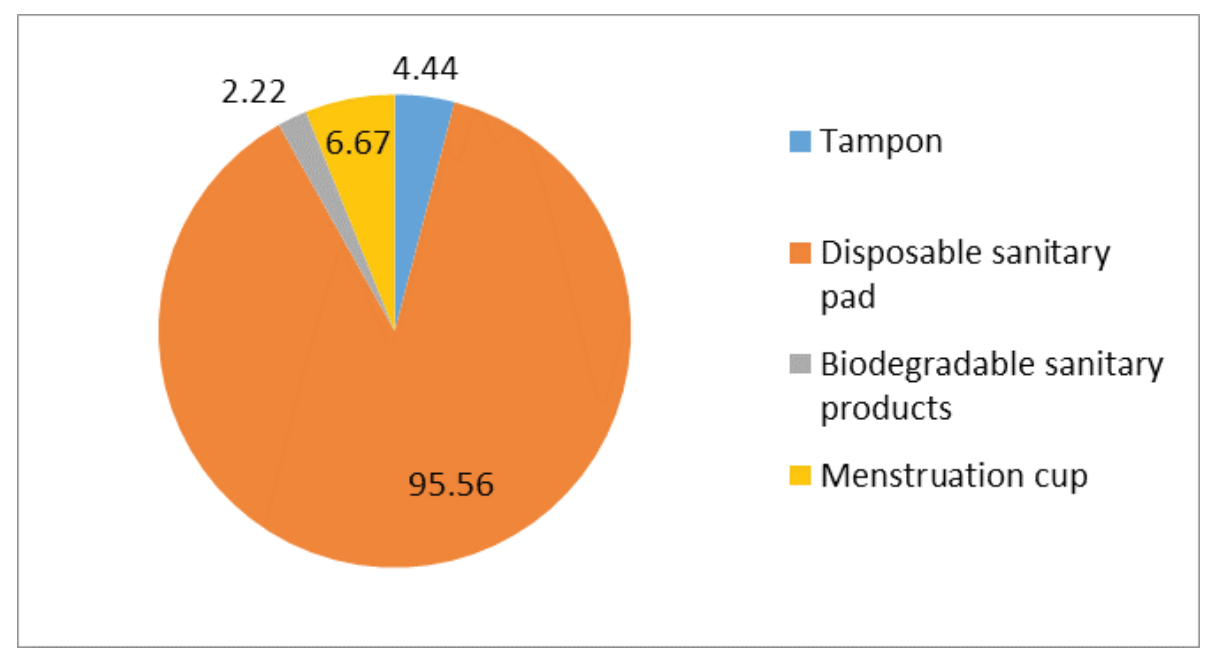

Source:Primary Data collected using structured questionnaire 
Sanitary protection products normally lead to different health problems like birth defects and infertility and also lead to environmental problems.

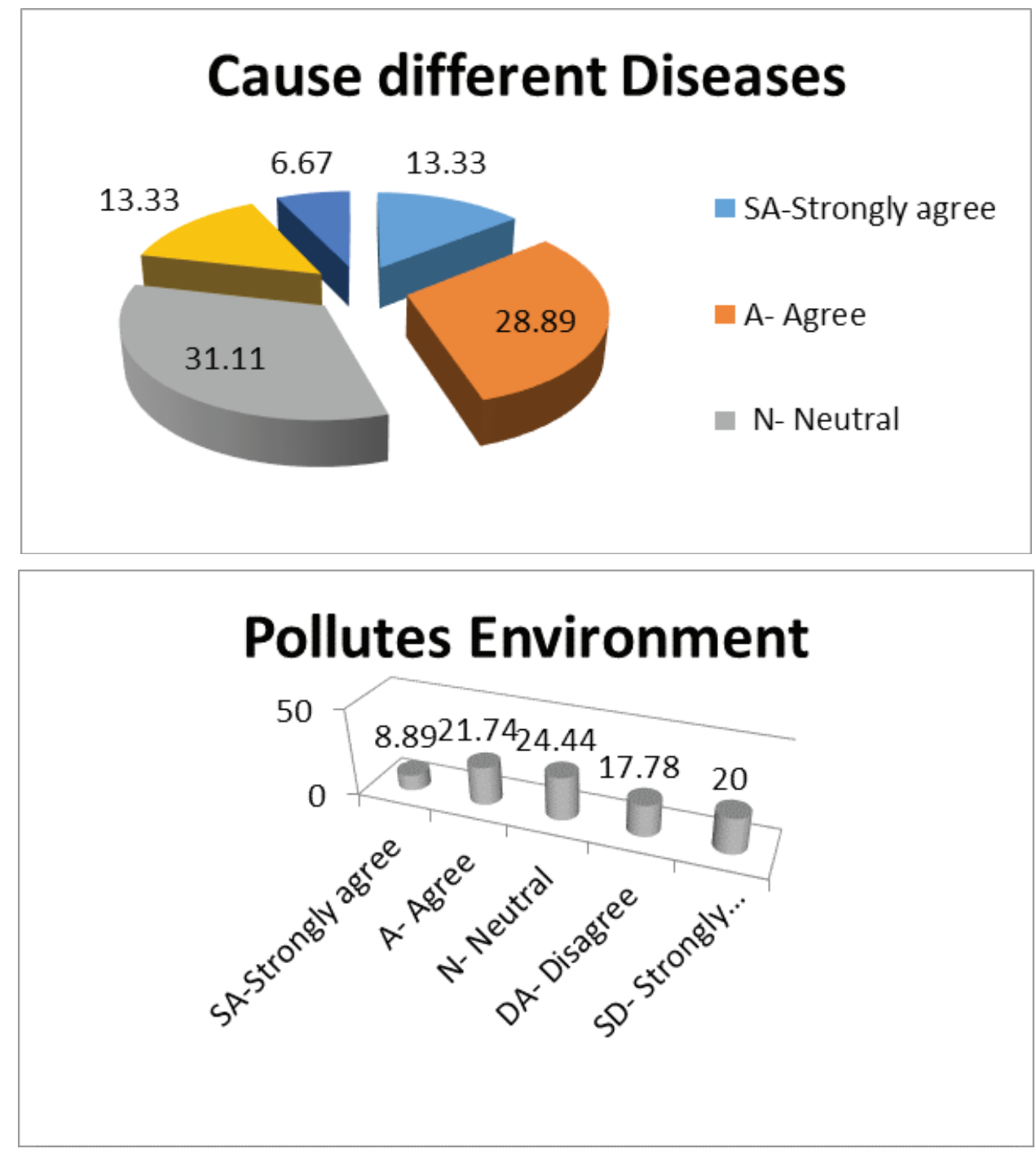

Source:Primary Data collected using structured questionnaire

$48.89 \%$ are agreed that ecofriendly products will address the women hygiene waste management problems.

As per the survey awareness at the grassroots level both in terms of use \& disposal and also about the hazards of careless disposal of pads will reduce the problem.

$88.89 \%$ respondents are looking for sustainable women hygiene product, but $33.33 \%$ are not aware about menstruation cup as an alternative product.Among $66.67 \%$ of the respondents (who are aware about the menstrual cups) $40 \%$ are agreeing that this product is safe without any side effects. $42.22 \%$ are of the view that menstruation cups are more practical, cheaper and ecofriendly. $22.22 \%$ strongly agree that usage of menstrual cups will reduce solid waste.

\section{Comparative analysis}

Comparative analysis of all three clusters selected for the research showed the following results. Among the 45 respondents selected from students, house wives and working women, $100 \%$ students are looking for a sustainable environmental friendly menstruation practices. $93.3 \%$ of the house wives and $80 \%$ of working 
women are looking out for sustainable menstruation practices.

Awareness about Menstruation cup as an alternative to sanitary protection product

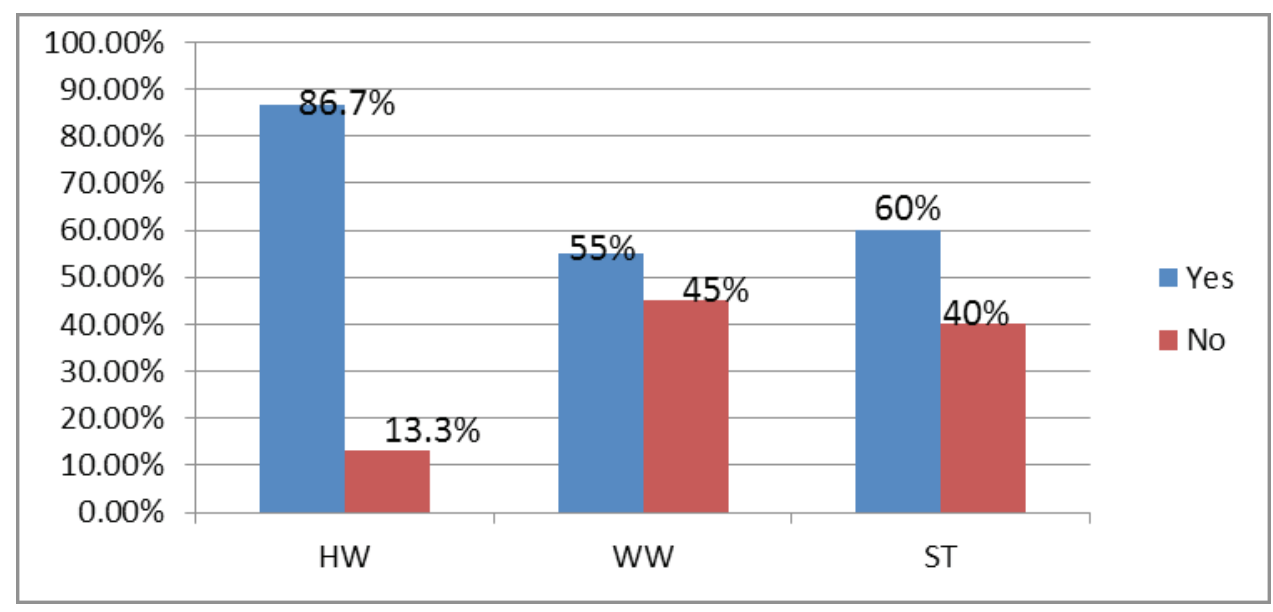

(HW-House wives, WW-Working Women, ST-Students)

\section{Source:Primary Data collected using structured questionnaire}

$86.7 \%$ of the house wives, $55 \%$ of working women and $60 \%$ of the students are aware about menstruation cup. Thus the research analysis clearly shows that, among the three clusters, students and house wives are looking out for sustainable menstruation practices and are aware about menstruation cup as an alternative solution.

Findings

Participants consider symptoms of PMS like pain, discomfort mood swings etc; are normal during menstrual cycles.

- Participants are not aware or negligent on treatment available for menstrual health problem.

- $21.4 \%$ uses technology driven apps to track their cycles and related problems.

Among the respondents $35.7 \%$ undergo yearly health checkups and $17.9 \%$ have never done full body checkups.

All respondents showed symptoms of Thyroid, but only $2.7 \%$ respondents have undergone Thyroid screening.

. Among the women hygienic products, disposable pads are commonly used and the users are aware about the health and environmental problems of using these products.

$51.1 \%$ agreed that Sorting, resorting and dumping of used pampers and sanitary pads lead to health hazards to waste collectors.

Many Nongovernmental organizations are initiated awareness towards sustainable women hygienic products among Rural Indian women.

- Research showed that, even though women are ready to use menstruation cup, they hesitate to use it because of lack of infrastructure like public toilet, water facilities etc;

- Insertion, removal and cleaning of menstruation cup are a commonly identified problem.

\section{Suggestions}

- Government and NGO's can conduct awareness campaign on menstrual hygiene and health issues.

Regular medical checkups and awareness campaign can be a part of public health programs.

Changed lifestyle, regular exercises, healthy 
diet, partner/family support, technology and mobile apps can solve the problems to a great extent.

Complicated problems of PMDD and thyroid have to be treated with proper medication by consulting medical practitioner.

Social, cultural and religious taboos on menstrual health problems can be avoided by changed education system and policies by the government.

Awareness about waste management is required from bottom to top level of the society.

Distribution and proper guidance of menstruation cup to the rural women may reduce the problem to a certain extent.

\section{Conclusion}

Today's Globalised fast moving world is creating numerous challenges to the people, especially to multi-tasking women. Lack of knowledge, awarenessofmenstrual disorders makes the problems complicated. Cultural, social and religious norms make it challenging to educate and communicate menstrual health in India.

Along with the increased menstrual health problems, sanitary waste management has become a serious issue. In order to prevent and minimize menstruation waste, we need sustainable female hygienic practices. The research shows that the women are willing to change from conventional to sustainable menstruation practices, but due to hesitation and lack of guidance, they are not coming for open discussion. Thus it is the duty of the society to make the women confident about the concept of menstruation and also to realize that it is not a shameful condemnation.

\section{Conflict of Interest statement}

The authors received moral support from CMS, Presidency Collegeand Royal Dental College. The present paper has been reviewed and accepted as working paper by Presidency CMS. The authors also conduct research in the area of Health Economics and public spending.
Source of Funding: The research was a self-funded project.

\section{Ethical Clearance Statement}

In undertaking this research various sources have been consulted in order to ensure that this study meets acceptable ethical guidelines. The paper is conceptual in nature and primary data obtained using structured questionnaire.

\section{References}

1. Bancroft J, Williamson L, Warner P, Rennie D, Smith SK. Perimenstrual complaints in women complaining of PMS, menorrhagia, and dysmenorrhea: toward a dismantling of the premenstrual syndrome. Psychosomatic Medicine. 1993 Mar.

2. Banerjee N, Roy KK, Takkar D. Premenstrual dysphoric disorder--a study from India. International journal of fertility and women's medicine. 2000 Sep 1;45(5):342-4.

3. Bohra N, Srivastava S, Bhatia MS. Depression in women in Indian context. Indian journal of psychiatry. 2015 Jul;57 (Suppl 2):S239.

4. Figert A. Women and the ownership of PMS: The structuring of a psychiatric disorder. Routledge; 2017 Sep 4.

5. Marjoribanks J, Brown J, O'Brien PM, Wyatt K. Selective serotonin reuptake inhibitors for premenstrual syndrome. Cochrane Database of Systematic Reviews. 2013(6).

6. Meenakshi N. Taboo in consumption: Social structure, gender and sustainable menstrual products. International Journal of Consumer Studies. 2020 May;44(3):243-57.

7. Murthy L. Green is the new colour for menstruation. Uger fabric pads show the way through a sustainable perspective. InICoRD'15-Research into Design Across Boundaries Volume 22015 (pp. 213-225). Springer, New Delhi.

8. O'Brien PS, Rapkin A, Schmidt PJ, editors. The premenstrual syndromes: PMS and PMDD. CRC Press; 2007 Oct 26.

9. Peberdy E, Jones A, Green D. A study into public awareness of the environmental impact of menstrual products and product choice. Sustainability. 2019 Jan;11(2):473. 
10. Raval CM, Panchal BN, Tiwari DS, Vala AU, Bhatt RB. Prevalence of premenstrual syndrome and premenstrual dysphoric disorder among college students of Bhavnagar, Gujarat. Indian Journal of psychiatry. $2016 \mathrm{Apr} ; 58(2): 164$.

11. Roe TE. Sustainability, menstrual products and sphagnum moss: an investigation (Doctoral dissertation, Lincoln University).
12. Ryu A, Kim TH. Premenstrual syndrome: A mini review. Maturitas. 2015 Dec 1;82(4):436-40.

13. Strine TW, Chapman DP, Ahluwalia IB. Menstrualrelated problems and psychological distress among women in the United States. Journal of women's health. 2005 May 1;14(4):316-23.

14. Taylor D. Effectiveness of professional-peer group treatment: symptom management for women with PMS. Research in Nursing \& Health. 1999 Dec;22(6):496-511. 